\title{
Prognostic significance of number of lymph node metastasis on survival in patients with pathological T3 esophageal carcinoma
}

\author{
K GULBEN ${ }^{1, *}$, F. IRKIN² ${ }^{2}$ M. YAZI ${ }^{3}$, U. BERBEROGLU ${ }^{1}$ \\ ${ }^{1}$ Ankara Oncology Education and Research Hospital, Department of Surgery, Ankara, Turkey; ${ }^{2}$ Letoon Private Hospital, Department of Surgery, \\ Fethiye, Mugla, Turkey; ${ }^{3}$ Cine Public Hospital, Department of Surgery, Cine, Aydin, Turkey
}

*Correspondence: kgulben@yahoo.com

Received July 13, 2016 / Accepted September 6, 2016

\begin{abstract}
The aim of this study was to evaluate the impact of the number of metastatic lymph nodes (MLN) and other risk factors on survival in patients with pathological T3 (pT3) esophageal carcinoma who were treated by esophagectomy. We analyzed 70 patients who received primary curative resection for pT3 esophageal cancer from 1997 to 2011 . The prognostic role of age, gender, tumor location, cell type, pathological lymph node status $(\mathrm{pN})$, number of MLNs ( $<3$ vs $\geq 3$ ), metastatic lymph node ratio (MLR), type of resection, local recurrence, and distant metastasis on overall survival (OS) were examined by univariate and multivariate analyses. Survival curves were calculated using Kaplan-Meier method and survival differences were assessed by log-rank test. A receiver operating characteristic analysis was used to determine the optimum cut-off point for the MLR. The median follow-up time was 42 (range, 8-128) months, and the 1-, 3- and 5-year OS rates were 78.6\%, 38.1\%, and $22.5 \%$, respectively. Tumor location, $\mathrm{pN}$, the number of MLNs, local recurrence, and distant metastasis had a significant effect on OS in the univariate analysis. In the multivariate model, the number of MLNs ( $p=0.02$; hazard ratio (HR), 2.1; 95\% confidence interval (CI), 1.1-4.1) and distant metastasis ( $\mathrm{p}=0.007$; HR, 5.1; 95\% CI, 1.5-16.8) were independent risk factors for OS. Patients with pT3 esophageal cancer who have 3 or more MLNs and distant metastasis have a poor OS, and this result can be used as a factor for better estimation of prognosis.
\end{abstract}

Key words: esophageal carcinoma, esophageal resection, lymph node, prognostic factors, survival

Esophageal cancer (EC) is the eighth most common cancer worldwide and the sixth most common cause of death from cancer [1]. ECs are histologically classified as squamous cell carcinoma (SCC) or adenocarcinoma [2]. SCC is the most common histology in Eastern Europa and Asia, and adenocarcinoma is most common in North America and most Western European countries [3]. Despite advances in early diagnosis and multidisciplinary treatment, patients with EC have still a poor prognosis and surgical resection remains the modality of choice for patients without systemic metastasis [4].

In published studies, several prognostic factors have been defined for survival in EC, such as age, gender, tumor location, tumor depth, lymph node (LN) status, cell type, number of metastatic lymph nodes (MLN), number of LNs removed, metastatic lymph node ratio (MLR), histologic differentiation, lymphovascular invasion $[1,3,5-8]$. The 7 th edition of the American Joint Committee on Cancer (AJCC) Staging Manual suggests the LN status to be the most significant risk factor in EC and categorizes $\mathrm{N}$ stage according to the number of MLNs [9]. According to this guideline for EC, pN1, pN2, and $\mathrm{pN} 3$ refer to 1-2, 3-6 and 7 or more positive lymph nodes, respectively. There is still controversy regarding the prognostic factors to be considered after esophagectomy for EC.

The goal of this study was to analyze retrospectively the role of number of MLNs and other clinicopathologic factors on overall survival (OS) in a homogeneous cohort of patients who had pathological T3 (pT3) EC treated with esophagectomy.

\section{Patients and methods}

Patients characteristics. The records of 136 patients with EC who were treated by esophagectomy were retrospectively evaluated in a single center between May 1997 and December 2011. The inclusion criteria were diagnosis of biopsy-proven carcinoma by endoscopy; pT3 tumor; no distant metastatic spread; no primary radiotherapy or chemotherapy; and 
histologically proven R0 resection. Sixty-six of 136 patients who did not meet the study criteria were excluded as well as patients who died during hospitalization or within the 30th postoperative day, as their deaths were considered not to be directly related to tumor staging. In addition, patients with gastroesophageal junction carcinoma were also excluded. The records of remaining 70 patients who met the above criteria were retrospectively evaluated. Institutional Review Board approval was obtained for the study.

Patients' treatment. All patients underwent a preoperative upper endoscopy with biopsy to confirm the diagnosis. CT-scan was the major diagnostic study to identify the local extent of the tumor and distant metastasis. Surgical procedures were subtotal Ivor-Lewis esophagectomy with proximal gastric resection through a right thoracotomy and transhiatal esophagectomy. Reconstruction consisted of a gastric tube placed through the posterior mediastinum, and esophagogastrostomy was performed intrathoracically or cervically. All patients had a peritumoral lymphadenectomy including dissection of the nodes in direct proximity to the tumor, the esophagus, and the upper stomach. In the majority of patients with tumor located lower third of esophagus, a standart dissection of perigastric, left gastric, and celiac nodes was also performed, but no extended lymphadenectomy was done. Macroscopic tumor clearance was achieved in all cases. Tumor staging for all patients in the study was performed according to the 7th edition of the AJCC Staging Manual [9]. Patients who had histopathologically proven to have MLNs received adjuvant chemotherapy and radiotherapy.

Postoperative patients were followed by physical examination every 3 months for the first year, every 3 to 6 months for the second year, and 6 to 12 months thereafter. Imaging studies which are computed tomographic scan and esophagoscopy were performed at regular intervals.

Patient demographics, tumor location, cell type, $\mathrm{pN}$ status, number of MLNs, MLR, type of resection, local recurrence, and distant metastasis were data recorded. To eveluate the

Table 1. Clinical and pathological characteristics of patients $(n=70)$

\begin{tabular}{llcc}
\hline Characteristics & variable & $\mathrm{n}$ & $\%$ \\
\hline Age $(\mathrm{yr})$ & $(\leq 50 />50)$ & $35 / 35$ & $50 / 50$ \\
Gender & (male / female) & $33 / 37$ & $47 / 53$ \\
Tumor location & (upper / middle / lower) & $8 / 8 / 54$ & $12 / 12 / 76$ \\
Type of resection & (transhiatal / Ivor Lewis) & $31 / 39$ & $44 / 56$ \\
Cell type & (SCC / adeno Ca) & $59 / 11$ & $84 / 16$ \\
pN & (negative/ positive) & $19 / 51$ & $27 / 73$ \\
No. of MLNs & $(<3 / \geq 3)$ & $32 / 19$ & $63 / 37$ \\
Adjuvant chemotherapy & (no / yes) & $25 / 45$ & $36 / 64$ \\
Adjuvant radiotherapy & (no / yes) & $9 / 61$ & $13 / 87$ \\
Local recurrence & (no / yes) & $55 / 15$ & $79 / 21$ \\
Distant metastasis & (no / yes) & $16 / 54$ & $23 / 77$ \\
\hline
\end{tabular}

$\mathrm{n}$, number of patients; SCC, squamous cell carcinoma; $\mathrm{pN}$, patological node status; MLN, metastatic lymph node relationship between the number of positive nodes and survival, the number of metastatic lymph nodes was subdivided into $\mathrm{pN} 1$ and $\mathrm{pN} 2$ plus $\mathrm{pN} 3$ ( $<3$ and $\geq 3 \mathrm{MLNs}$ ) based on the 7 th edition of the AJCC Staging Manual (9). The values of MLR was obtained on the basis of the number of positive nodes divided by the total number LNs removed. OS was estimated from the date of surgery to the date of death or last follow-up.

Statistical analysis. All statistical tests were carried out using the SPSS version 17.0 for Windows (SPSS Inc., Chicago, IL, USA). The OS was calculated using Kaplan-Meier method, and the difference was evaluated by the log-rank test. Univariate and multivariate analyses were performed by Cox regression. A receiver operating characteristic (ROC) analysis with the area under the curve (AUC) was used to determine the optimum cut-off point for the MLR. All statistical tests of significance were two-sided, and a p value less than 0.05 was considered to be statistically significant.

\section{Results}

The median age was 50 years (range, 18 - 80 years) and the median follow-up time was 42 months (range, 8-128 months). Clinicopathologic characteristics of 70 pT3 EC patients who received primary curative resection are summerized in Table 1. Of 70 patients, 37 (53\%) were female, and 33 (47\%) were male. Histologically, 59 patients had SCC (84\%), and 11 patients had adenocarcinoma (16\%). Tumor location was detected to be frequently in lower third (76\%) of the esophagus. Ivor-Lewis procedure was performed in 39 patients $(56 \%)$ and remaining 31 patients (44\%) underwent transhiatal resection. The median number of LNs removed was 12 (range, 3-48) and a total of 51 patients (73\%) had MLN in their pathological specimens. The median number of MLNs was 2 (range, 1-19) and the numbers of patients with $\mathrm{pN} 1$ and $\mathrm{pN} 2$ plus $\mathrm{pN} 3$ were 32 (63\%) and $19(37 \%)$, respectively. Of 70 patients, 22 had stage 2 EC (31\%) and 48 had stage 3 EC (69\%) according to TNM classification. During the follow-up period, 15 patients $(21 \%)$ developed local recurrence and 54 patients (77\%) developed distant metastasis. Fifty-eight patients died from progression of the disease. The estimated 1-, 3- and 5-year OS rates were $78.6 \%, 38.1 \%$, and $22.5 \%$, respectively.

The optimal cut-off point for MLR was identified as 0.13 by ROC analysis (AUC, $0.61 ; \mathrm{p}=0.23$; 95\% CI, $0.43-0.79$ ) and the numbers of patients who had $<0.13$ of MLR and $\geq 0.13$ were $32(46 \%)$ and 38 (54\%), respectively. Univariate analysis results are presented in Table 2. Tumor location, $\mathrm{pN}$ status, the number of MLNs, local recurrence, and distant metastasis were significant risk factors for OS ( $\mathrm{p}<0.04$ for all variables). However, age, gender, type of resection, cell type, and MLR had not significant effect on OS ( $>>0.05)$. The factors that were found to be significant in the univariate analysis were included in the multivariate analysis (Table 3). In that model, we demonstrated that the number of MLNs $(<3 \mathrm{vs.} \geq 3)$ was independent prognostic factor for OS ( $\mathrm{p}=0.02 ; \mathrm{HR}, 2.1 ; 95 \%$ 
CI, 1.1-4.1). In Kaplan-Meier survival estimation, the 5-year OS rate in patients with $<3$ MLNs and $\geq 3$ MLNs were $17 \%$ (median 34 months) and 5\% (median 18 months), respectively $(\mathrm{p}=0.01$, Figure 1). Additionally, distant metastasis was also found as the other significant risk factor for OS in the multivariate analysis ( $\mathrm{p}=0.009$; HR, 5.1; 95\% CI, 1.5-16.8).

\section{Discussion}

In spite of the recent practice of incorporating chemotherapy and chemoradiotherapy into the treatment of EC, surgical resection remains the cornerstone for early and locally advanced disease [10]. Over the past three decades, with developments in surgical techniques and perioperative care, surgical mortality from esophageal resection has decreased consistently. In this study, we collected a well-defined homogeneous cohort of 70 patients with pT3 EC and assessed the outcomes to determine prognostic factors.

Our study showed that the age, gender, type of resection, cell type, and MLR had not significant effect on OS. Although the pathologic characteristics of the primary tumor including tumor location, $\mathrm{pN}$ status, the number of MLNs, local recurrence, and distant metastasis were significant risk factors for OS in the univariate analysis, the number of MLNs and distant metastsis were correlate with outcome in the multivariable model.

Survival is affected not only by the presence or absence of positive lymph nodes (pN0 vs $\mathrm{pN} 1$ ) but also the number of MLNs in patients with EC [3]. Published data reported that the number of MLNs for EC is an important prognostic marker without distinguishing adenocarcinoma from squamous type $[5,11,12]$. The current AJCC staging system for esophageal cancer underlines the importance of depth of invasion ( $\mathrm{T}$ ) and the number of involved lymph nodes. In the presented study, patients who had 1-2 MLNs in their resection specimens had a better OS compared to those with $\geq 3$ MLNs. This study also indicated that a staging considering the number of involved lymph nodes improves the prognostic

Table 2. Univariate analysis of factors affecting overall survival.

\begin{tabular}{llccc}
\hline Characteristics & Variable & $P$-value & HR & $95 \%$ CI \\
\hline Age $(\mathrm{yr})$ & $(>50$ vs $\leq 50)$ & NS & - & - \\
Gender & (male vs female) & NS & - & - \\
Tumor location & (lower vs upper/middle) & 0.04 & 1.9 & $1.05-3.7$ \\
Type of resection & $($ transhiatal vs Ivor Lewis) & NS & - & - \\
Cell type & $($ SCC vs adeno ca) & NS & - & - \\
pN & $($ positive vs negative) & 0.04 & 1.9 & $1.02-3.6$ \\
No. of MLNs & $(\geq 3$ vs $<3)$ & 0.01 & 2.1 & $1.1-3.9$ \\
MLR & $(\geq 0.13$ vs $<0.13)$ & NS & - & - \\
Local recurrence & $($ yes vs no $)$ & 0.03 & 1.8 & $1.03-3.4$ \\
Distant metastasis & $($ yes vs no) & 0.001 & 5.6 & $2.02-15.5$ \\
\hline
\end{tabular}

SCC, squamous cell carcinoma; $\mathrm{pN}$, pathological node status; MLN, metastatic lymph node; MLR, metastatic lymph node ratio; HR, hazard ratio; CI, confidence interval; NS, not significant

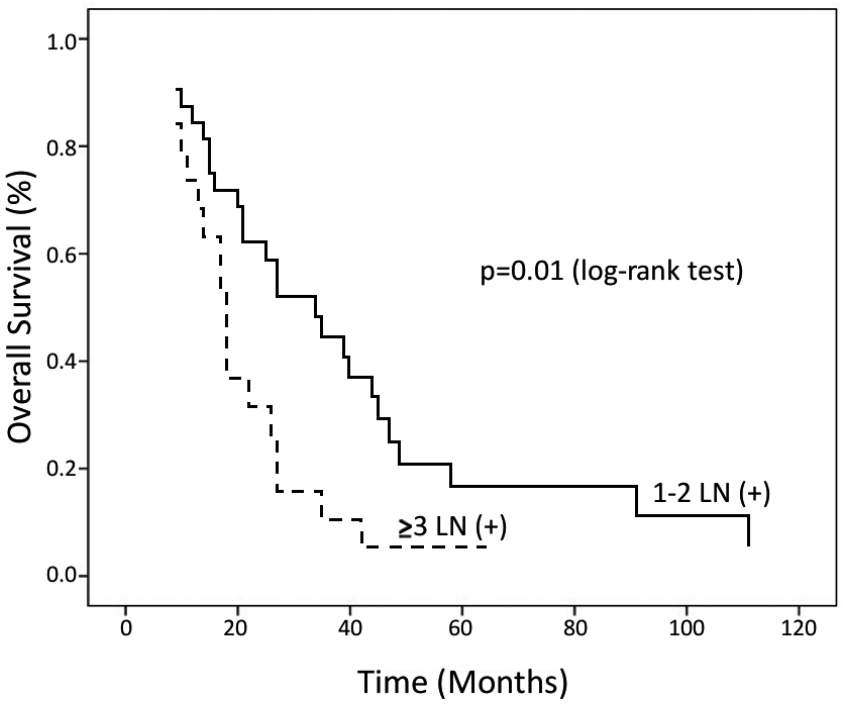

Figure 1. Overall survival reveals statistically significant differences between patients with 1-2 $\mathrm{LN}(+)$ and $\geq 3 \mathrm{LN}(+)$.

power compared with the N0-N1 criterion. A significant reduction of OS for patients with equal to or more than three positive nodes was determined. The 5 -year OS rate in patients with $<3$ MLNs and $\geq 3$ MLNs were $17 \%$ and 5\%, respectively. Based on the seventh edition AJCC staging system, the 5-year OS rates were $37 \%, 14 \%, 5.3 \%$, and $3.4 \%$ in patients classified as having stage $\mathrm{pN} 0, \mathrm{pN} 1, \mathrm{pN} 2$, and $\mathrm{pN} 3$, respectively $[8,9]$. These survival rates are similar with our results. Furthermore, our results are similar to studies presented by Rice et al [13] and Li et al [3]. They subclassified the node positive patients as N1 ( $\leq 2$ MLNs) and N2 ( $\geq 3$ MLNs) on the basis of significantly different survival rates. Other studies also showed that patients with more advanced nodal disease had worse survival than those with less nodal disease [14, 15]. In our study, the correlation between the number of MLNs and survival may be explained with the increasing in tumor burden of disease and, therefore, to predict outcome. This result proposes that more aggressive adjuvant therapy may play the key role to improve the survival of patients with multiple lymph node metastasis.

Table 3. Multivariate analysis of factors affecting overall survival

\begin{tabular}{llccc}
\hline Characteristics & Variable & $P$-value & HR & $95 \%$ CI \\
\hline Tumor location & (lower vs upper/middle) & NS & & \\
pN & (positive vs negative) & NS & & \\
No. of MLNs & $(\geq 3$ vs $<3$ ) & 0.02 & 2.1 & $1.1-4.1$ \\
Local recurrence & (yes vs no) & NS & & \\
Distant metastasis & (yes vs no) & 0.009 & 5.1 & $1.5-16.8$ \\
\hline
\end{tabular}

SCC, squamous cell carcinoma; $\mathrm{pN}$, pathological node status; MLN, metastatic lymph node; MLR, metastatic lymph node ratio; HR, hazard ratio; CI, confidence interval; NS, not significant. 
Within the group of patients with MLNs, an other subdivision can be composed on the basis of the number of positive nodes divided by the total number of LNs removed, also known as the MLR [3, 8, 16, 17]. Various MLRs have been proposed due to differences in the techniques of lymph node dissection and the total number of LNs removed. In patients including this study, lymphadenectomies consists of the peritumoral lymphadenectomy with dissection of the nodes in direct proximity to the tumor, the esophagus, and the upper stomach. In the majority of patients with tumor located lower third of esophagus, a standart dissection of perigastric, left gastric, and celiac nodes was also performed. It has been reported that in patients undergoing esophagectomy without induction chemoradiation, at least 15 lymph nodes should be removed to achieve adequate nodal staging [18]. The median number of lymph nodes removed in our study was relatively low (a median number of 12) and probably relates to the high percentage of transhiatal resections (44\%). Another reason of this might be that all patients did not undergo an extended lymphadenectomy. Our study suggests that the MLR was not an independent predictive factor for OS because it was not associated with differences in survival. Other studies also reported that the MLR was not a prognostic factor for OS in patients with EC $[3,19]$. Nevertheless, more studies are needed to verify this result.

The other clinical variable independently predicting OS was distant metastasis in the current study. Five-year OS in patients who had no distant metastasis and those with distant metastasis were $71 \%$ and $13 \%$, respectively. During the followup period, 54 patients (77\%) developed distant metastasis. Distant disease is still a poor prognostic factor even in patients underwent esophageal resection with curative intent for EC. New systemic treatment agents are needed for improving outcomes in this clinical setting. In the presented study, the estimated 1-, 3- and 5-year OS rates were 78.6\%, 38.1\%, and $22.5 \%$, respectively. These survival rates are similar with other reports $[8,20]$.

In conclusion, this study shows that the long term results are significantly correlated with the number of involved lymph nodes. Patients with 1 to 2 metastatic nodes after radical esophagectomy have a better prognosis than those with 3 or more involved nodes. Furthermore, distant metastasis is the other poor prognostic factor after resection of EC. Consideration of these two factors can help in the selection of adjuvant therapy which could improve survival outcomes.

\section{References}

[1] JEMAL A, BRAY F, CENTER MM, FERLAY J, WARD E et al. Global cancer statistics. CA Cancer J Clin 2011; 61: 69-90. https:/doi.org/10.3322/caac.20107

[2] SIEWERT JR, OTT K. Are squamous and adenocarcinomas of the esophagus the same disease? Semin Radiat Oncol 2007; 17: 38-44. https:/doi.org/10.1016/j. semradonc.2006.09.007
[3] LI H, ZHANG Q, XU L, CHEN Y, WEI Y et al. Factors predictive of prognosis after esophagectomy for squamous cell cancer. J Thorac Cardiovasc Surg 2009; 137: 55-59. https:/doi. org/10.1016/j.jtcvs.2008.05.024

[4] FENG JF, ZHAO Q, CHEN QX. Prognostic value of subcarinal lymph node metastasis in patients with esophageal squamous cell carcinoma. Asian Pac J Cancer Prev 2013; 14: 3183-3186. https:/doi.org/10.7314/APJCP.2013.14.5.3183

[5] RIZK N, VENKATRAMAN E, PARK B, FLORES R, BAINS MS et al. The prognostic importance of the number of involved lymph nodes in esophageal cancer: implications for revisions of the American Joint Committee on Cancer staging system. J Thorac Cardiovasc Surg 2006; 132: 1374-1381. https:/doi. org/10.1016/j.jtcvs.2006.07.039

[6] PEYRE CG, HAGEN JA, DEMEESTER SR, ALTORKI NK, ANCONA E et al. The number of lymph nodes removed predicts survival in esophageal cancer: an international study on the impact of extent of surgical resection. Ann Surg 2008; 248: 549-556. https:/doi.org/10.1097/ sla.0b013e318188c474

[7] IELPO B, PERNAUTE AS, ELIA S, BUONOMO OC, VALLADARES LD et al. Impact of number and site of lymph node invasion on survival of adenocarcinoma of esophagogastric junction. Interact Cardiovasc Thorac Surg 2010; 10: 704-708. https:/doi.org/10.1510/icvts.2009.222778

[8] HE Z, WU S, LI Q, LIN Q, XU J. Use of the metastatic lymph node ratio to evaluate the prognosis of esophageal cancer patients with node metastasis following radical esophagectomy. PLoS One 2013; 8: e73446. https:/doi.org/10.1371/journal. pone.0073446

[9] RICE TW, BLACKSTONE EH, RUSCH VW. 7th edition of the AJCC Cancer Staging Manual: esophagus and esophagogastric junction. Ann Surg Oncol 2010; 17: 1721-1724. https:/doi. org/10.1245/s10434-010-1024-1

[10] DeMEESTER SR. Adenocarcinoma of the esophagus and cardia: a review of the disease and its treatment. Ann Surg Oncol 2006; 13: 12-30. https:/doi.org/10.1245/ $\underline{\text { ASO.2005.12.025 }}$

[11] BOLLSCHWEILER E, BALDUS SE, SCHRODER W, SCHNEIDER PM, HOLSCHER AH. Staging of esophageal carcinoma: length of tumor and number of involved regional lymph nodes. Are these independent prognostic factors? J Surg Oncol 2006; 94: 355-363. https:/doi.org/10.1002/ jso. 20569

[12] PETERS CJ, HARDWICK RH, VOWLER SL, FITZGERALD RC. Generation and validation of a revised classification for oesophageal and junctional adenocarcinoma. Br J Surg 2009; 96: 724-733. https:/doi.org/10.1002/bjs.6584

[13] RICE TW, BLACKSTONE EH, RYBICKI LA, ADELSTEIN DJ, MURTHY SC et al. Refining esophageal cancer staging. J Thorac Cardiovasc Surg 2003; 125: 1103-1113. https:/doi. org/10.1067/mtc.2003.170

[14] WU N, CHEN Z, PANG L, MA Q, CHEN G. Prognostic significance of lymph node characteristics on survival in esophageal squamous cell carcinomas. Wien Klin Wochenschr 2013; 125: 26-33. https:/doi.org/10.1007/s00508-012$\underline{0310-2}$ 
[15] AKUTSU Y, MATSUBARA H. The significance of lymph node status as a prognostic factor for esophageal cancer. Surg Today 2011; 41: 1190-1195. https:/doi.org/10.1007/s00595011-4542-y

[16] SAHA S, DEHN TC. Ratio of invaded to removed lymph nodes as a prognostic factor in adenocarcinoma of the distal esophagus and esophagogastric junction. Dis Esophagus 2001; 14: 32-36. https:/doi.org/10.1111/j.14422050.2001.00148.x

[17] ELOUBEIDI MA, DESMOND R, ARGUEDAS MR, REED CE, WILCOX CM. Prognostic factors for the survival of patients with esophageal carcinoma in the U.S.: the importance of tumor length and lymph node status. Cancer 2002; 95: 1434-1443. https:/doi.org/10.1002/cncr.10868
[18] RIZK NP, ISHWARAN H, RICE TW, CHEN LQ, SCHIPPER PH et al. Optimum lymphadenectomy for esophageal cancer. Ann Surg 2010; 251: 46-50. https:/doi.org/10.1097/ SLA.0b013e3181b2f6ee

[19] WILSON M, ROSATO EL, CHOJNACKI KA, CHERVONEVA I, KAIRYS JC et al. Prognostic significance of lymph node metastases and ratio in esophageal cancer. J Surg Res 2008; 146: 11-15. https:/doi.org/10.1016/j.jss.2007.07.028

[20] WIJNHOVEN BP, TRAN KT, ESTERMAN A, WATSON DI, TILANUS HW. An evaluation of prognostic factors and tumor staging of resected carcinoma of the esophagus. Ann Surg 2007; 245: 717-725. https:/doi.org/10.1097/01. $\underline{\text { sla.0000251703.35919.02 }}$ 